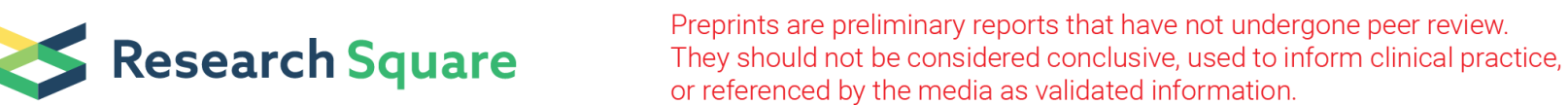

\section{Development and Evaluation of Quality of Life Instruments for Chronic Diseases-Chronic Pulmonary Heart Disease (QLICD-CPHD) (V2.0)}

Haiyan Pan

Guangdong Medical University

Zheng Yang

Guangdong Medical University

Chonghua Wan ( $\nabla$ wanchh@hotmail.com )

Guangdong Medical University

Jinghao Ruan

Fifth Affiliated Hospital of Sun Yat-sen University

Bin Wu

Guangdong Medical University

$\mathrm{Na} \mathrm{Li}$

The first affiliated hospital of kunming medical university

\section{Xingjie Wang}

Guangdong Medical University

\section{Zhihuan Zhao}

The first affiliated hospital of kunming medical university

\section{Research}

Keywords: Chronic pulmonary heart disease, Quality of life, Instrument, Reliability, Validity,

Responsiveness

Posted Date: February 27th, 2020

DOl: https://doi.org/10.21203/rs.2.24759/v1

License: (c) (i) This work is licensed under a Creative Commons Attribution 4.0 International License.

Read Full License 


\section{Abstract}

Objective: To develop a quality of life (QOL) instrument specific to chronic pulmonary heart disease (CPHD) and to evaluate its reliability, validity, and responsiveness.

Methods: Under the Quality of Life Instruments for Chronic Diseases (QLICD) system, the QLICD-CPHD (V2.0) was developed and used to measure the QOL of 184 patients with CPHD. Data were analyzed by statistical description, linear correlation analysis, exploratory factor analysis, and paired t-test.

Results: The QLICD-CPHD (V2.0) consisted of 44 items, including 28 in the general module and 16 in the specific module. The Cronbach's a coefficients of four domains in the general module and the specific module were greater than 0.7 , and the split-half reliability coefficients were between 0.6 and 0.8 , indicating that the instrument has good reliability. The instrument showed good validity as the correlation coefficients among items of the same domain were significantly higher than those among items of different domains. Correlation coefficients $r$ between the corresponding domains of SF-36 and QLICDCPHD were between 0.26 and 0.60 , which suggests that QLICD-CPHD has moderate criterion validity. Paired t-tests of scores of patients before and after treatment showed significant differences in the overall score, scores of general module domains except social role, and scores of the specific module. Standardized response means were greater than 0.2 , suggesting that the instrument had moderate responsiveness and was sensitive to changes in the patients' QOL.

Conclusion: The QLICD-CPHD (V2.0) has good reliability, validity, and responsiveness. The instrument can be used to evaluate the QOL of CPHD patients.

\section{Introduction}

Chronic pulmonary heart disease (CPHD) is caused by pulmonary vascular resistance arising from chronic pathological changes in the pulmonary thoracic space or pulmonary arteries, which result in pulmonary hypertension and right ventricular hypertrophy with or without right heart failure ${ }^{[1]}$. CPHD is common in China, with a reported mean incidence of $0.41-0.47 \%{ }^{[2]}$. As a chronic respiratory disease, $\mathrm{CPHD}$ is characterized by a long course, high recurrence rate, need for intervention, and irreversible damage, which make it difficult to evaluate treatment efficacy on the basis of a cure rate. Therefore, the health status of patients is often determined by objective clinical indicators such as blood gas analysis and pulmonary function tests ${ }^{[3-4]}$. At present, a human-centered, subjective instrument known as patientreported outcomes (PROs) has been developed to measure individual health status. PROs are evaluation indicators developed based on quality of life (QOL) and specifically reflect the health status of patients ${ }^{[5]}$. Therefore, it is difficult to separate PROs from QOL. To improve the accuracy of the QOL instrument, evaluation of the QOL of CPHD patients must include patients' feelings about life and health and must reflect the impacts the disease has on the physical, psychological, and social activities of patients in their daily life. 
Survey instruments are an important tool for the evaluation of the QOL of CPHD patients. Most instruments that have been used in recent years to measure the QOL of CPHD patients are generic, such as the Nottingham Health Profile (NHP) ${ }^{[6-7]}$ and the 36-Item Short Form Health Survey Questionnaire (SF$36)^{[8-9]}$. Although specific instruments for many conditions are commonly used in clinical practice, a specific instrument for CPHD is still lacking. In addition, the existing instruments do not take into account the characteristics of the Chinese populations, values, and cultures, and thus may not be suitable for Chinese patients ${ }^{[10]}$. Clearly, it is inappropriate to apply instruments from other countries without modification. Therefore, it is necessary to develop a CPHD-specific instrument with Chinese characteristics to evaluate of the QOL of CPHD patients and clinical efficacy of treatments. A QOL instrument for chronic diseases-CPHD (QLICD-CPHD) developed by Wan ${ }^{[5]}$ in China contains four domains, namely, physical functions, psychological functions, social functions, and specific functions. However, there are still some inadequacies in its application, so the instrument needs to be modified and improved.

This study is therefore aimed at developing an improved instrument with Chinese characteristics and evaluating its reliability, validity, and responsiveness.

\section{Materials And Methods 2.1 Subjects}

The subjects of this study were 184 patients with clinically confirmed CPHD who were hospitalized in the First Affiliated Hospital of Kunming Medical University and the Second Affiliated Hospital of Kunming Medical University. The inclusion criteria were CPHD patients who were able to read and write and volunteered to participate in the evaluation.

\subsection{Methods}

On the basis of literature including the existing QLICD-CPHD ${ }^{[19]}$ and the QLICD process, the QLICD-CPHD (V2.0), a self-assessed questionnaire for Chinese patients, was developed by combining a general module for chronic diseases (QLICD-GM) with a specific module for $\mathrm{CPHD}^{[11-12]}$. The resultant instrument consists of 44 items, 28 of which are in the general module and 16 of which are in the specific module. The general module consists of physical functions ( 9 items), psychological functions (11 items), and social functions (8 items). The specific module includes six facets, namely cough and expectoration, lung deficiency, heart failure, pulmonary encephalopathy, oxygenation, and influence on life by specific psychology.

The subjects were first given a brief explanation, and then after their consent was obtained, the QLICDCPHD (V2.0) was distributed to them, and they filled it out according to their actual conditions. A questionnaire was completed both on the first day of admission and on the day of discharge.

\subsection{Statistical analysis}


Data were analyzed using SPSS 22.0. The main statistical methods used were statistical description, correlation analysis, paired t-test, exploratory factor analysis, one-way analysis of variance, and multiple linear regression analysis. Unless otherwise specified, all P values were two-tailed, and an a value of 0.05 was considered statistically significant.

\subsection{Scoring method}

Each item is scored using a five-point Likert scale, which includes "not at all" (1 point), "a little" (2 points), "more or less" (3 points), "quite a bit" (4 points), and "very" (5 points) as the response options. The instrument consists of both positive and negative items. Higher scores for the positive items and lower scores for the negative items indicate better QOL for patients.

The raw score (RS) of each domain and facet as well as the total score were calculated. The RS of a domain/facet was the sum of the scores of items in this domain/facet. In order to compare the scores among domains, range standardization was used to convert RS into a standard score (SS) $(0-100)^{[9]}$ according to the formula

$S S=\left(R S-S_{\min }\right) \times 100 /\left(S_{\max }-S_{\min }\right)$,

where $S_{\min }$ (minimum score) represents the possible minimum score of a domain/facet and $S_{\max }$ (maximum score) represents the possible maximum score of a domain/facet.

\section{Results}

\subsection{General characteristics of patients}

All 184 patients involved in this study completed the survey at admission and upon discharge. The specific demographic characteristics of the CPHD patients are shown in Table 1. The majority of patients were males (77.2\%), of Han nationality, and married. The mean age of the patients was 45 years, and the dominant occupations were worker and civil servant, of which worker accounted for the largest proportion (35.9\%). For education level, a plurality of patients were primary school graduates (37.5\%), followed by middle school (26.6\%) and high school/technical secondary school (23.9\%). The primary form of medical insurance was social medical insurance (medical insurance for urban workers) (80.4\%), followed by cooperative medical insurance (23.5\%). Most patients' financial status was moderate (73.4\%).

\subsection{Scale reliability}

The internal consistency and split-half reliability of the four domains in the general module and the specific module of QLICD-CPHD are shown in Table 2. The Cronbach's a of all domains in the instrument were greater than 0.738 , and the Cronbach's a of the entire instrument was 0.899 , which indicate high internal consistency. The split-half reliability of the general module domains and specific module ranged between 0.612 and 0.795 , and the split-half reliability of the entire instrument was 0.795 . Some 
researchers recommended a Cronbach's a of 0.6 as the minimum acceptable value ${ }^{[15]}$. Overall, the instrument had moderate internal consistency.

\subsection{Instrument validity}

\subsubsection{Item-dimension correlation}

Correlation analysis was performed for each item and domain of the instrument. The results showed that the correlation coefficients were high for most of the QLICD-CPHD domains. Correlations were significantly higher between items and their respective domains than between items and other domains. This suggests that items and dimensions of QLICD-CPHD are highly correlated, and the instrument design was practical and reasonable (Table 3).

\subsubsection{Exploratory factor analysis}

Bartlett's test of sphericity showed correlations among items in the specific module of QLICD-CPHD and a Kaiser-Meyer-Olkin statistic of 0.796 , indicating very weak partial correlation. Thus, an exploratory factor analysis was performed on the data using principal component analysis. Factors with eigenvalues greater than 1 were extracted and converted by varimax rotation. The items and item factor loadings for each principal component are shown in Table 4 and Fig. 1.

A total of four principal components were extracted from the specific module score of QLICD-CPHD (V2.0) by factor analysis, and the cumulative variance contribution rate was as high as $62.20 \%$. The first principal component included items CPHD6, CPHD7, CPHD8, and CPHD9, and the cumulative variance contribution rate was $30.76 \%$. This component consisted of the items for lung deficiency (LDF). The second principal component included items CPHD1, CPHD2, CPHD3, and CPHD4, and the cumulative variance contribution rate was $12.18 \%$. This component consisted of the items for cough and expectoration (CAP). The third principal component included items CPHD5, CPHD10, CPHD11, and CPHD16, and the cumulative variance contribution rate was $11.13 \%$. The fourth principal component included items CPHD12, CPHD13, CPHD14, and CPHD15, and the cumulative variance contribution was $8.13 \%$. The third and fourth principal components reflected heart failure (HEF), pulmonary encephalopathy (PUE), oxygenation (OXT), and influence on life by specific psychology (ILS). The structure of the specific module was largely consistent with the theoretical construct, which should consist of four facets. Therefore, the principal components obtained by maximum variance factor rotation were generally consistent with the theoretical construct, which indicates moderate validity of the specific module of the instrument.

\subsubsection{Criterion validity}

The SF-36 instrument was used as a validity criterion due to the lack of a gold standard. Correlation analysis of the corresponding domains of QLICD-CPHD (V2.0) and SF-36 showed that the corresponding 
domains of the two instruments had higher correlation coefficients than noncorresponding domains, which indicates that QLICD-CPHD has a good criterion validity (Table 5).

\subsection{Instrument responsiveness}

In this study, patients were asked to fill out the instrument at admission and upon discharge, and the total score as well as domain and facet scores of the instrument were analyzed using a paired $t$-test and standardized response mean (SRM). The mean scores of each domain/facet and the mean total scores of the first and second assessment were calculated and analyzed by a paired $t$-test. The SRM, which is the absolute value of the ratio of the difference before and after treatment to its standard deviation, was calculated.

The paired $t$-test showed that the total scores were significantly different before and after treatment $(\mathrm{P}<$ some very small number). Aside from social role, other domain/facet scores were significantly different after treatment. The SRM of social role in the general module was low, while the SRM values of other domains/facets were greater than 0.2 , which indicates that the instrument scores were significantly changed after treatment (Table 6). These findings indicate that the instrument is sensitive to the changes in the patients' QOL and is highly responsive.Changes of mean value in five fields before and after treatment(Fig 2).which indicates that the mean value was significantly changed after treatment.

\section{Discussion}

This study was conducted within the framework of a research project on QLICD ${ }^{[12]}$. The instrument was developed by combining a general module and a specific module, which is a promising approach of instrument development. Programmed instrument development procedures ensure good content validity of the instrument. The coexistence of the general module and specific module help incorporate the characteristics of traditional Chinese cultures, dietary habits, and family factors into the scope of the instrument ${ }^{[13-14]}$.

Most patients showed a high level of questionnaire completion, and the recovery rate and passing rate of the questionnaire were $100 \%$, suggesting high feasibility and acceptability.

\subsection{Reliability}

Reliability is a measure of the precision of the instrument. We used homogeneity reliability and split-half reliability to evaluate the internal stability of the instrument. The higher the reliability coefficients, the higher the reliability of the instrument or facets. A Cronbach's a of 0.6 was previously recommended as the minimum acceptable value ${ }^{[15]}$. We found that the Cronbach's a of QLICD-CPHD (V2.0) modules were greater than 0.738 , which is an indication of good reliability. The overall internal consistency and overall split-half reliability of the instrument were 0.899 and 0.795 , respectively. In addition, the Cronbach's a and split-half reliability of most domains/facets were greater than 0.6 . 


\subsection{Validity}

Validity is a measure of the accuracy of the instrument. The higher the validity of an instrument, the more accurate the characteristics it measures.

The validity of QLICD-CPHD (V2.0) was evaluated by research validity and criterion validity. Criterion validity measures the correlation between an instrument and a criterion by calculating the correlation coefficients of their scores. We used SF-36 as the criterion and showed that the domains of QLICD-CPHD (V2.0) had good correlations with the corresponding domains of SF-36, indicating that the instrument has a good criterion validity.

Item-dimension and exploratory factor analyses showed that the specific module of QLICD-CPHD was generally consistent with its theoretical construct. Some items were not partitioned into the corresponding principal components as described by the theoretical instrument, which may be attributable to the interrelatedness of symptoms. In addition, the small sample size in this study may also affect the results. Nevertheless, we can still argue that the measured results were generally congruent with the concept of the instrument and the instrument has a good structural validity.

\subsection{Responsiveness}

Responsiveness reflects the QOL of patients in different treatment phases and is useful for comparing the efficacy of different treatments. Responsiveness is the most important indicator in the study of instrument application, and it is directly related to the evaluation and selection of treatment regimes. Our results revealed that aside from social role, the total scores as well as the domain scores of the QLICDCPHD general module and specific module were significantly different before and after treatment, and all SRM values were greater than 0.2 . This suggests that the instrument has a moderate responsiveness. Conditions of patients that were directly improved after admission and targeted treatment were mainly reflected in the disease-specific domain. The responsiveness to treatment in hospital can vary according to the nature of different diseases. This instrument can sensitively reflect the changes in the QOL of patients during hospitalization with a certain degree of responsiveness.

\subsection{Limitations}

This study has some limitations. First, the sample size used was relatively small. Second, the test-retest reliability of the instrument was not measured. Third, the selected subjects were all inpatients, which can lead to selection bias as patients with mild symptoms or patients who were not hospitalized due to financial difficulties were not included in the survey. Finally, the comprehensiveness of the instrument could not be evaluated due to the lack of follow-up studies of the discharged patients.

\section{Conclusions}

The QLICD-CPHD (V2.0) consists of 44 items, 28 of which are in the general module and 16 of which are in the specific module. Use of the instrument to measure the QOL of $184 \mathrm{CPHD}$ patients showed that its 
reliability, validity, and responsiveness were good. Furthermore, the instrument has better sensitivity and specificity than the SF-36, which is a generic instrument commonly used worldwide ${ }^{[16-19]}$. The QLICDCPHD (V2.0) can be used as a tool to measure the QOL of CPHD patients and provide a basis for the evaluation of clinical treatment efficacy and the selection of treatment strategies.

\section{Abbreviations}

QLICD-CPHD

quality of life instruments for chronic diseases in chronic pulmonary heart disease.

PROs

patient-reported outcomes.

NHP

Nottingham Health Profile.

QOL

Quality of life.

SF-36

the MOS item short from health survey, SF-36.

SRM

The standard responsive mean.

RS

Raw score.

SS

Standardized score.

ANOVA

one-way analysis of variance.

KMO

Kaiser-Meyer-Olkin.

CVCR

cumulative variance contribution rate.

CGD

Core/General domain.

PHD

Physical domain.

PSD

Psychological domain.

SOD

Social domain.

SPD

Chronic Prostatitis Special Domain. 


\section{Declarations}

\section{Ethics approval and consent to participate:}

All procedures performed in studies involving human participants were in accordance with the ethical standards of the institutional and/or national research committee and with the 1964 Helsinki declaration and its later amendments or comparable ethical standards.

\section{Consent for publication}

Not applicable.

\section{Availability of data and material}

The datasets used and/or analyzed during the current study are available from the corresponding author on reasonable request.

\section{conflict of interest}

The authors declared that they have no conflicts of interest to this work.

\section{Funding}

Supported by National Natural Science Foundation of China.

\section{Authors' contributions}

HYP participated in the design of this study and wrote the manuscript. CHW and Zheng Yang carried out the study and collected important background information. XJW and Jinghao Ruan processed the data. Bin Wu, Na Li and Zhihuan Zhao collected the data.

\section{Acknowledgements}

In carrying out this research project, we have received substantial assistance from Wu Li, YupingYang $\rrbracket$ Guohui Wang at the first affiliated hospital of Kunming Medical University and Min Chen, Jun Wu, Dongming Li at the affiliated hospital of Guangdong Medical University. We sincerely acknowledge all the support.

\section{Authors' information}

HYP,Associate Professor, Master Tutor, School of Public Health, Guangdong Medical University.ZY,School of Humanities and Management, Research Center for Quality of Life and Applied Psychology, Guangdong Medical University. CHW, Professor, Doctoral Tutor, School of Public Health, Guangdong Medical University. JHR, The Fifth Affiliated Hospital of Sun Yat-sen University, Zhuhai. BW,The Affiliated Hospital of Guangdong Medical University, Zhanjiang .NL,The First Affiliated Hospital of Kunming Medical 
University, Kunming.XJW,Graduate Student of Guangdong Medical University. ZHZ,The First Affiliated Hospital of Kunming Medical University, Kunming.

\section{References}

[1] Zheng, Q.C. Analysis of 76 cases of chronic pulmonary heart disease [J]. Chinese Journal of Coal Industry Medicine, 2010,13(12), 1786-1787.

[2] Mei, X.M., \& Zhang, X.R. Progress in diagnosis and treatment of chronic pulmonary heart disease [J]. Gems of Health, 2012,11(7), 532-532.

[3] Li, N., Zhao Z.H., \& Wan, C.H. Research on the influencing factors for living quality of patients with chronic pulmonary heart disease [J] Journal of Qiqihar Medical University, 2010,31(4), 513-514.

[4] Li, N., Zhao, Z.H., \& Wan, C.H. Relationships between quality of life and clinical indicators of patients with chronic pulmonary heart disease [J] Journal of Jilin University (Medicine Edition),2012, 38(3), 580585 .

[5] Wang, G.H., Li, X.M., Wan, C.H., et al. . Development and evaluation of quality of life instrument for patients with chronic pulmonary heart disease [J]. Chinese Journal of Public Health, 2009,25(10), 12241226.

[6] Ramírez-Vélez, R. Quality of life and social support for patients suffering from chronic obstructive pulmonary disease [J]. Rev Salud Publica (Bogota), 2007,9(4), 568-575.

[7] Shafazand S, Goldstein MK, Doyle RL, et al. Health-related quality of life in patients with pulmonary arterial hypertension [ J ]. Chest, 2004, 126(5), 1452-1459.

[8] Karapolat H, Eyigor S, Atasever A, et al. Effect of dyspnea and clinical variables on the quality of life and functional capacity in patients with chronic obstructive pulmonary disease and congestive heart failure [J]. Chinese Medical Journal (English), 2008, 121(7), 592-596.

[9] Mereles D, Ehlken N, Kreuscher S, et al. Exercise and respiratory training improve exercise capacity and quality of life in patients with severe chronic pulmonary hypertension[J]. Circulation, 2006, 114(14), 1482-1489.

[10] Ulrich S, Speich R, Domenighetti G, et al. Bosentan therapy for chronic thromboembolic pulmonary hypertension. A national open label study assessing the effect of Bosentan on haemodynamics, exercise capacity, quality of life, safety and tolerability in patients with chronic thromboembolic pulmonary hypertension (BOCTEPH-Study) [J]. Swiss Medical Weekly, 2007,137(41-42), 573-580.

[11] Wan C, Tu X, Messing S, et al. Development and validation of the general module of the system of quality of life instruments for chronic diseases and its comparison with SF-36[J]. Journal of Pain \& Symptom Management, 2011, 42(1), 93-104. 
[12] Wan $\mathrm{CH}$. Measurement and assessment of quality of life [M]. Kunming: Yunnan University Press, 1999, 1-90.

[13] Yang Z, Qi YB, Wan $\mathrm{CH}$, et al. Using item response theory to analyze general module of quality of life instruments for chronic diseases [J]. Chinese General Practice, 2012, 15 (22), 2544-2547.

[14] Yanchun S, Zheng Y, Chonghua W, et al. Development and validation of the pulmonary tuberculosis scale of the system of Quality of Life Instruments for Chronic Diseases (QLICD-PT). Health and Quality of Life Outcomes, 2018, 16(1), 137-[last page number].

[15] Samsa G, Matchar DB, Dolor RJ, et al. A new instrument for measuring anticoagulation-related quality of life: development and preliminary validation[J]. Health Qual Life Outcomes, 2004, 2(1), 22.

[16] Wan $\mathrm{CH}$, Zhao XD, Li XM, et al. Comparison of two instruments on measuring quality of life for patients with chronic diseases [J]. Chinese Journal of Public Health, 2007, 23(7), 815-817.

[17] Wu J, Hu L, Zhang G, et al. Development and validation of the nasopharyngeal cancer scale among the system of quality of life instruments for cancer patients (QLICP-NA V2.0): combined classical test theory and generalizability theory[J]. Quality of Life Research, 2016, 25(8), 2087-2100.

[18] Xiaohui R, Chaojie L, Ningxiu L. Assessing the Quality of Life of People with Chronic Diseases Using SF-36[J]. Journal of West China University of Medical Sciences, 2001, 32(2), 250-253.

[19] Guan YX, Jin SD, Lu N, et al. Hope level and social support status of patients with chronic pulmonary heart disease and their correlation [J]. Practical Journal of Cardiac Cerebral Pneumal and Vascular Disease, 2015 (1), 92-94.

\section{Tables}

Table 1 General information of CPHD patients 


\begin{tabular}{|c|c|c|c|c|c|c|c|}
\hline Items & Category & $\begin{array}{l}\text { Number } \\
\text { of } \\
\text { cases }\end{array}$ & $\begin{array}{l}\text { Percentage } \\
(\%)\end{array}$ & Items & Category & $\begin{array}{l}\text { Number } \\
\text { of } \\
\text { cases }\end{array}$ & $\begin{array}{l}\text { Percentage } \\
(\%)\end{array}$ \\
\hline \multirow[t]{4}{*}{ Gender } & Male & 142 & 77.2 & Nationality & Han & 176 & 95.7 \\
\hline & Female & 42 & 22.8 & & Others & 8 & 4.3 \\
\hline & Worker & 66 & 35.9 & & Unmarried & 2 & 1.1 \\
\hline & Farmer & 33 & 17.9 & & Married & 148 & 80.4 \\
\hline \multirow[t]{5}{*}{ Occupation } & Teacher & 11 & 6.0 & Marital & Divorced & 1 & 0.5 \\
\hline & Civil servant & 43 & 23.4 & & Widowed & 33 & 17.9 \\
\hline & $\begin{array}{l}\text { Self-employed and } \\
\text { others }\end{array}$ & 31 & 16.8 & & & & \\
\hline & Primary school & 69 & 37.5 & & Self-paid & 8 & 4.3 \\
\hline & Middle school & 49 & 26.6 & & Commercial insurance & 3 & 1.6 \\
\hline \multirow{6}{*}{$\begin{array}{l}\text { Education } \\
\text { level }\end{array}$} & High school or & 44 & 23.9 & Medical & Cooperative & 25 & 13.6 \\
\hline & technical secondary & & & insurance & insurance & & \\
\hline & school & & & & & & \\
\hline & Junior college & 13 & 7.1 & & Social medical insurance & 148 & 80.4 \\
\hline & $\begin{array}{l}\text { Undergraduate and } \\
\text { above }\end{array}$ & 9 & 4.9 & & $\begin{array}{l}\text { (medical insurance for } \\
\text { urban workers) }\end{array}$ & & \\
\hline & Low & 44 & 23.9 & & & & \\
\hline \multirow{3}{*}{$\begin{array}{l}\text { Economic } \\
\text { status }\end{array}$} & Moderate & 135 & 73.4 & Age & $45.0 \pm 49.0$ & & \\
\hline & High & 5 & 2.7 & & & & \\
\hline & $\mathrm{N}$ & 736 & & $\mathrm{~N}$ & 552 & & \\
\hline
\end{tabular}

Notes: N:Total number of cases. 
Table 2 Internal consistency coefficient and split-half reliability of QLICD-CPHD

\begin{tabular}{|c|c|c|}
\hline Domains/Facets & Cronbach's a & Split-half reliability \\
\hline Physical domain (PHD) & 0.738 & 0.612 \\
\hline Basic physical functions (BPF) & 0.766 & 0.766 \\
\hline Independence (IND) & 0.849 & 0.884 \\
\hline Energy and discomfort (EAD) & 0.433 & 0.516 \\
\hline Psychological domain (PSD) & 0.826 & 0.795 \\
\hline Cognition (COG) & 0.344 & 0.318 \\
\hline Emotion (EMO) & 0.832 & 0.737 \\
\hline Will and personality (WIP) & 0.430 & 0.431 \\
\hline Social domain (SOD) & 0.744 & 0.654 \\
\hline Interpersonal communication (INC) & 0.640 & 0.719 \\
\hline Social support and security (SSS) & 0.444 & 0.450 \\
\hline Social role (SOR) & 0.517 & 0.322 \\
\hline Core/General domain (CGD) & 0.887 & 0.772 \\
\hline Special domain (SPD) & 0.806 & 0.659 \\
\hline Cough and expectoration (CAP) & 0.793 & 0.740 \\
\hline Lung deficiency (LDF) & 0.847 & 0.870 \\
\hline Heart failure (HEF) & 0.471 & 0.473 \\
\hline Total (TOT) & 0.899 & 0.795 \\
\hline
\end{tabular}

*N=184(Sample Size). 
Table 3 Correlations between item and domain scores of QLICD-CPHD 


\begin{tabular}{|c|c|c|c|c|}
\hline Items & Physical functions & Psychological functions & Social functions & Specific functions \\
\hline GPH1 & 0.60 & 0.28 & 0.16 & 0.24 \\
\hline GPH2 & 0.51 & 0.25 & 0.10 & 0.16 \\
\hline GPH3 & 0.33 & 0.13 & 0.11 & 0.03 \\
\hline GPH4 & 0.39 & 0.17 & 0.05 & 0.20 \\
\hline GPH5 & 0.44 & 0.29 & 0.11 & 0.24 \\
\hline GPH6 & 0.77 & 0.38 & 0.29 & 0.33 \\
\hline GPH7 & 0.68 & 0.29 & 0.28 & 0.23 \\
\hline GPH8 & 0.78 & 0.35 & 0.36 & 0.31 \\
\hline GPH9 & 0.53 & 0.36 & 0.26 & 0.37 \\
\hline GPS1 & 0.48 & 0.54 & 0.45 & 0.20 \\
\hline GPS2 & 0.16 & 0.41 & 0.06 & 0.36 \\
\hline GPS3 & 0.10 & 0.38 & 0.34 & -0.09 \\
\hline GPS4 & 0.18 & 0.63 & 0.35 & 0.38 \\
\hline GPS5 & 0.36 & 0.66 & 0.49 & 0.28 \\
\hline GPS6 & 0.31 & 0.57 & 0.35 & 0.13 \\
\hline GPS7 & 0.36 & 0.76 & 0.43 & 0.42 \\
\hline GPS8 & 0.31 & 0.78 & 0.49 & 0.41 \\
\hline GPS9 & 0.44 & 0.80 & 0.55 & 0.34 \\
\hline GPS10 & 0.43 & 0.59 & 0.61 & 0.16 \\
\hline GPS11 & 0.25 & 0.69 & 0.41 & 0.38 \\
\hline GS01 & 0.34 & 0.45 & 0.65 & 0.19 \\
\hline GSO2 & 0.13 & 0.34 & 0.62 & 0.14 \\
\hline
\end{tabular}




\begin{tabular}{|c|c|c|c|c|}
\hline GSO3 & -0.01 & 0.24 & 0.56 & -0.08 \\
\hline GSO4 & 0.10 & 0.51 & 0.76 & 0.23 \\
\hline GSO5 & 0.13 & 0.44 & 0.78 & 0.09 \\
\hline GSO6 & 0.37 & 0.35 & 0.40 & 0.19 \\
\hline GSO7 & 0.32 & 0.43 & 0.46 & 0.20 \\
\hline GSO8 & 0.21 & 0.38 & 0.66 & 0.06 \\
\hline CPHD1 & 0.31 & 0.29 & 0.13 & 0.55 \\
\hline CPHD2 & 0.14 & 0.35 & 0.13 & 0.63 \\
\hline CPHD3 & 0.16 & 0.25 & 0.12 & 0.46 \\
\hline CPHD4 & 0.15 & 0.21 & 0.07 & 0.60 \\
\hline CPHD5 & 0.33 & 0.31 & 0.12 & 0.70 \\
\hline CPHD6 & 0.32 & 0.16 & 0.13 & 0.59 \\
\hline CPHD7 & 0.21 & 0.27 & 0.13 & 0.65 \\
\hline CPHD8 & 0.38 & 0.33 & 0.19 & 0.63 \\
\hline CPHD9 & 0.40 & 0.30 & 0.16 & 0.58 \\
\hline CPHD10 & 0.14 & 0.21 & 0.06 & 0.62 \\
\hline CPHD11 & 0.12 & 0.21 & 0.09 & 0.52 \\
\hline CPHD12 & 0.28 & 0.14 & 0.13 & 0.43 \\
\hline CPHD13 & 0.12 & 0.14 & 0.14 & 0.46 \\
\hline CPHD14 & 0.26 & 0.21 & 0.06 & 0.37 \\
\hline CPHD15 & 0.29 & 0.20 & 0.11 & 0.45 \\
\hline CPHD16 & -0.10 & 0.05 & 0.08 & -0.04 \\
\hline
\end{tabular}


Table 4 Factor loadings for items in the specific module of QLICD-CPHD (V2.0) after varimax rotation

\begin{tabular}{|c|c|c|c|c|}
\hline \multirow[t]{2}{*}{ Items } & \multicolumn{4}{|c|}{ Principal component (variance contribution rate, \%) } \\
\hline & $1(30.76)$ & $2(12.18)$ & $3(11.13)$ & $4(8.13)$ \\
\hline CPHD1 & & 0.79 & & \\
\hline CPHD2 & & 0.52 & & \\
\hline CPHD3 & & 0.75 & & \\
\hline CPHD4 & & 0.86 & & \\
\hline CPHD5 & & & 0.55 & \\
\hline CPHD6 & 0.77 & & & \\
\hline CPHD7 & 0.75 & & & \\
\hline CPHD8 & 0.85 & & & \\
\hline CPHD9 & 0.85 & & & \\
\hline CPHD10 & & & 0.66 & \\
\hline CPHD11 & & & 0.81 & \\
\hline CPHD12 & & & & 0.40 \\
\hline CPHD13 & & & & 0.73 \\
\hline CPHD14 & & & & 0.48 \\
\hline CPHD15 & & & & 0.74 \\
\hline CPHD16 & & & 0.47 & \\
\hline
\end{tabular}

Note: Values less than 0.4 are not reported. 
Table 5 Correlation between QLICD-CPHD (V2.0) domains and SF-36 items

\begin{tabular}{|c|c|c|c|c|c|c|c|c|}
\hline \multirow[t]{2}{*}{ SF-36 } & \multicolumn{8}{|c|}{ QLICD-CPHD } \\
\hline & $\begin{array}{l}\text { Physical } \\
\text { (PHD) }\end{array}$ & domain & $\begin{array}{l}\text { Psychological } \\
\text { (PSD) }\end{array}$ & domain & $\begin{array}{l}\text { Social } \\
\text { (SOD) }\end{array}$ & domain & $\begin{array}{l}\text { Specific } \\
\text { (SPD) }\end{array}$ & domain \\
\hline $\begin{array}{l}\text { Physical functioning } \\
\text { (PF) }\end{array}$ & 0.54 & & 0.20 & & 0.19 & & 0.39 & \\
\hline Role physical (RP) & 0.29 & & 0.20 & & $0.12^{\star}$ & & 0.24 & \\
\hline Bodily pain (BP) & 0.55 & & 0.45 & & 0.29 & & 0.51 & \\
\hline General health $(\mathrm{GH})$ & 0.26 & & $-0.03^{\star}$ & & $-0.02^{\star}$ & & $0.04^{*}$ & \\
\hline Vitality (VT) & 0.60 & & 0.29 & & 0.24 & & 0.22 & \\
\hline Social functions (SF) & 0.50 & & 0.29 & & 0.27 & & 0.32 & \\
\hline Role emotional (RE) & 0.27 & & 0.34 & & 0.19 & & 0.23 & \\
\hline Mental health $(\mathrm{MH})$ & 0.32 & & 0.58 & & 0.44 & & 0.20 & \\
\hline
\end{tabular}

Note: * indicates no significant difference $(P>0.05)$

Table 6 Comparison of scores of various QLICD-CPHD (V2.0) domains before and after treatment 


\begin{tabular}{|c|c|c|c|c|c|c|c|c|c|}
\hline \multirow{2}{*}{$\begin{array}{l}\text { Domains/ } \\
\text { Facets }\end{array}$} & \multicolumn{2}{|c|}{ Before treatment } & \multicolumn{2}{|c|}{ After treatment } & \multicolumn{2}{|c|}{ Difference } & \multicolumn{2}{|c|}{ Paired $t$-test } & \multirow[t]{2}{*}{ SRM } \\
\hline & Mean & $\begin{array}{l}\text { Standard } \\
\text { deviation }\end{array}$ & Mean & $\begin{array}{l}\text { Standard } \\
\text { deviation }\end{array}$ & Mean & $\begin{array}{l}\text { Standard } \\
\text { deviation }\end{array}$ & $t$ & $P$ & \\
\hline General module & 51.50 & 12.72 & 57.73 & 12.26 & -6.23 & 8.68 & -9.73 & 0.000 & 0.72 \\
\hline Physical domain & 42.95 & 14.52 & 51.92 & 13.66 & -8.97 & 10.40 & -11.70 & 0.000 & 0.86 \\
\hline Basic physical functions & 46.26 & 14.05 & 56.62 & 12.21 & -10.36 & 12.58 & -11.17 & 0.000 & 0.82 \\
\hline Independence & 42.84 & 26.17 & 47.74 & 26.20 & 4.89 & 14.56 & 4.56 & 0.000 & 0.34 \\
\hline Energy and discomfort & 36.48 & 21.47 & 48.78 & 20.46 & -12.30 & 20.35 & -8.20 & 0.000 & 0.60 \\
\hline Psychological domain & 51.73 & 16.54 & 58.35 & 16.07 & -6.62 & 11.98 & -7.50 & 0.000 & 0.55 \\
\hline Cognition & 49.59 & 18.84 & 54.96 & 18.39 & -5.37 & 17.46 & -4.17 & 0.000 & 0.31 \\
\hline Emotion & 51.71 & 18.58 & 58.73 & 18.42 & 7.03 & 13.48 & 7.07 & 0.000 & 0.52 \\
\hline Will and personality & 53.94 & 20.78 & 60.39 & 18.43 & -6.45 & 15.52 & -5.64 & 0.000 & 0.42 \\
\hline Social domain & 59.48 & 15.51 & 62.09 & 14.68 & -2.62 & 9.77 & -3.63 & 0.000 & 0.27 \\
\hline $\begin{array}{l}\text { Interpersonal } \\
\text { communication }\end{array}$ & 62.95 & 16.93 & 66.58 & 16.73 & -3.62 & 11.64 & -4.22 & 0.000 & 0.31 \\
\hline $\begin{array}{l}\text { Social support and } \\
\text { security }\end{array}$ & 60.55 & 19.83 & 63.18 & 18.99 & -2.63 & 13.09 & -2.72 & 0.007 & 0.20 \\
\hline Social role & 52.65 & 22.19 & 53.74 & 20.51 & -1.09 & 17.00 & -0.87 & 0.387 & 0.06 \\
\hline Specific module & 43.91 & 13.02 & 58.71 & 15.18 & -14.80 & 13.53 & -14.84 & 0.000 & 1.09 \\
\hline Cough and expectoration & 39.47 & 20.60 & 58.53 & 19.91 & -19.06 & 18.56 & -13.92 & 0.000 & 1.03 \\
\hline
\end{tabular}




\begin{tabular}{|c|c|c|c|c|c|c|c|c|c|}
\hline Pulmonary insufficiency & 32.25 & 17.89 & 46.22 & 20.22 & -13.97 & 17.56 & -10.79 & 0.000 & 0.80 \\
\hline Heart failure & 59.69 & 19.41 & 70.47 & 18.86 & -10.78 & 17.75 & -8.24 & 0.000 & 0.61 \\
\hline $\begin{array}{l}\text { Pulmonary } \\
\text { encephalopathy }\end{array}$ & 63.04 & 26.13 & 73.91 & 23.20 & -10.87 & 23.75 & -6.21 & 0.000 & 0.46 \\
\hline Oxygenation & 59.10 & 27.07 & 87.23 & 20.91 & -28.13 & 32.23 & -11.84 & 0.000 & 0.87 \\
\hline $\begin{array}{l}\text { Influence on life by } \\
\text { specific psychology }\end{array}$ & 50.00 & 26.91 & 55.43 & 25.83 & -5.43 & 21.18 & -3.48 & 0.001 & 0.26 \\
\hline Total & 48.99 & 11.08 & 58.20 & 11.57 & -9.21 & 9.23 & -13.54 & 0.000 & 1.00 \\
\hline
\end{tabular}

*N=184(Sample Size).

\section{Figures}




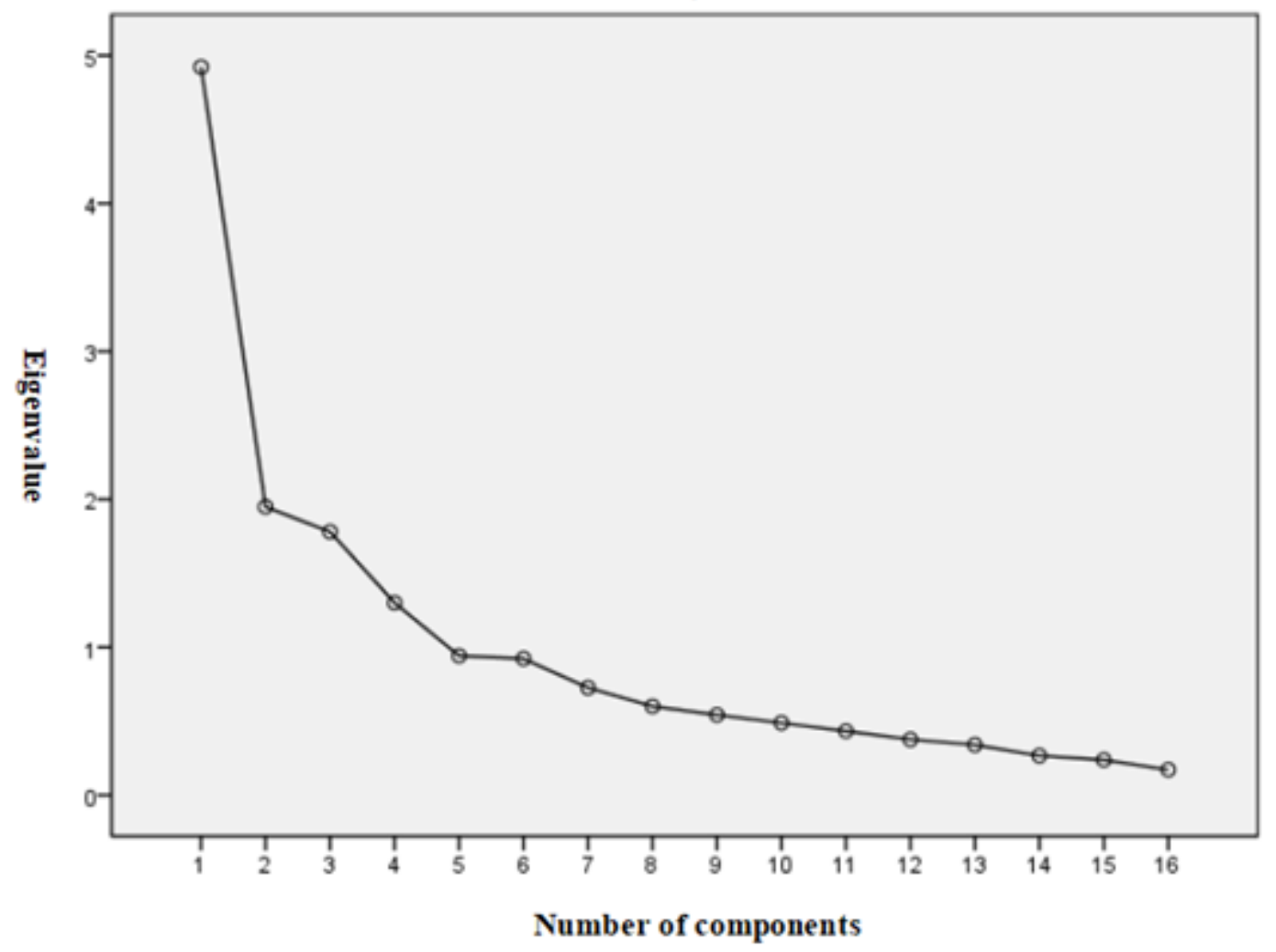

Figure 1

Scree plot of items in the specific module of QLICD-CPHD (V2.0)

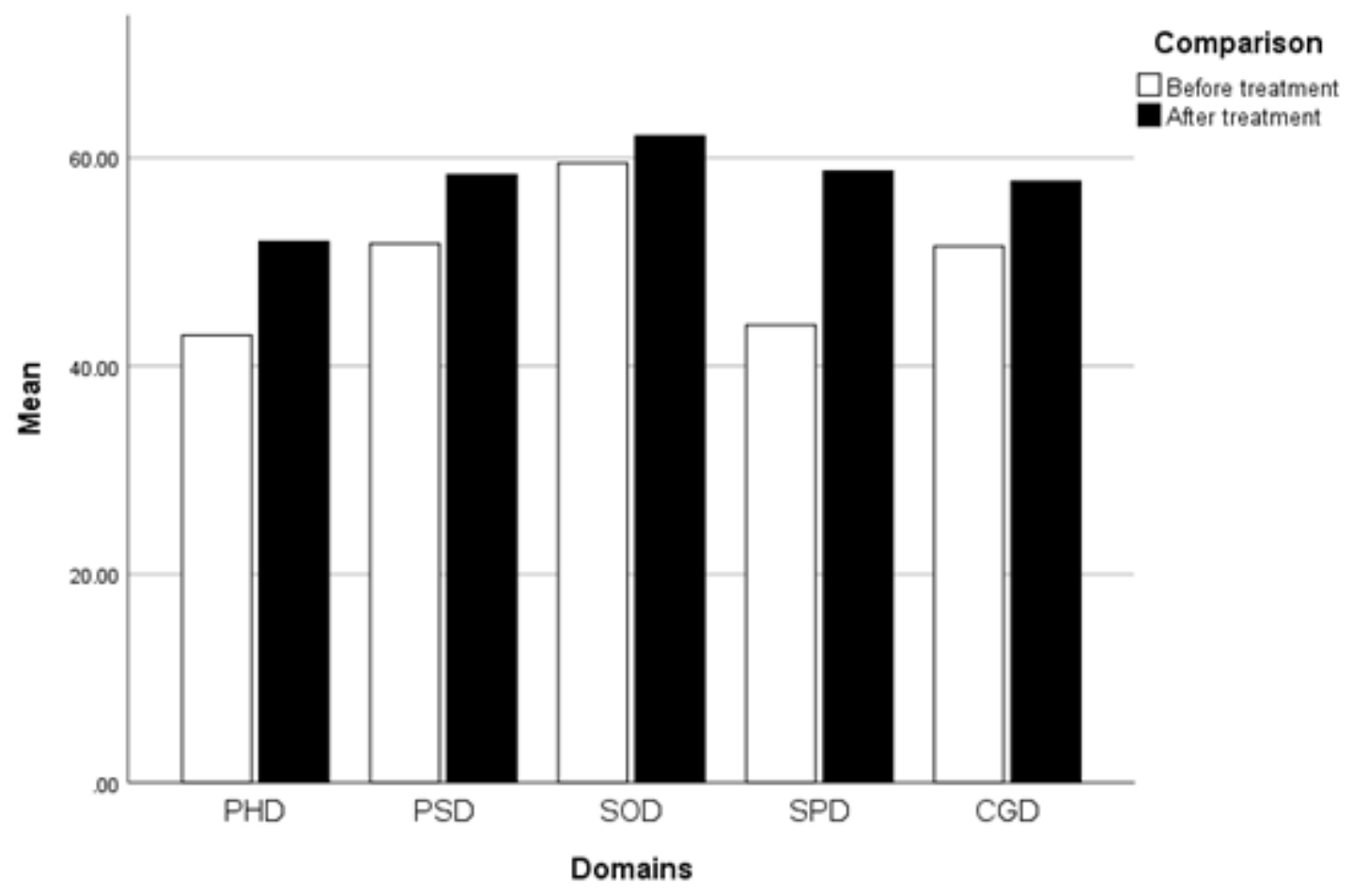


Figure 2

Mean value in five fields before and after treatment 